\title{
Facteurs explicatifs de la faible mobilisation des recettes du secteur minier dans la province du Haut-Katanga
}

\author{
Par MWENZE MINZA Martin*
}

\section{INTRODUCTION}

La province du Haut-Katanga figure parmi les vingt-six provinces de la République Démocratique du Congo. Cette province est incroyablement riche en ressources naturelles, notamment minières. Elle regorge d'importants gisements de cuivre à haute teneur et des minerais associés tels que le cobalt, le zinc, le plomb,....Cette situation explique la forte présence des entreprises minières devant engendrer les flux financiers conséquents pour le compte de cette province :

- Pour la Direction Générale des Impôts, DGI en sigle :

- L'impôt sur les bénéfices et profits (IBP);

- L'impôt professionnel sur les rémunérations (IPR);

- L'impôt exceptionnel sur la rémunération des expatriés (IER);

- L'impôt mobilier (IM);

- L'impôt sur le chiffre d'affaires à l'intérieur (ICAI).

- Pour la Direction Générale des Douanes et accises, DGDA en sigle :

- Le droit d'entrée (DE);

- La redevance et les frais en rémunération des services rendus à l'exportation (SRE);

- Les redevances administratives.

- Pour la Direction Générale des Recettes Administratives, Judiciaires, Domaniales et de Participations, DGRAD en sigle :

- Les dividendes versés à l'Etat;

- Les droits superficiaires annuels par carré;

- Les pas-de-porte versés à l'Etat;

- Les redevances minières $(\mathrm{RM})$.

- Pour la Direction des Recettes du Haut-Katanga, DRHKAT en sigle :

- La taxe provinciale incitative à la création des unités locales de traitement des concentrés;

- La taxe provinciale d'interventions en matière de réhabilitation des infrastructures urbaines de voirie et drainage ainsi que des routes d'intérêt provincial;

- L'impôt sur la superficie des concessions minières et des hydrocarbures.

* MWENZE MINZA Martin Chef de Travaux à l'UNILU Master en Sciences Politiques et Administratives. 
De 2008 à 2015, la province du Haut-Katanga a connu quelques recettes du secteur minier ci-dessous :

- Taxe provinciale incitative à la création des unités locales de traitement des concentrés : 31.692.852.972.245 Francs Congolais;

- Taxe provinciale d'interventions en matière de réhabilitation des infrastructures urbaines de voirie et drainage ainsi que des routes d'intérêt provincial : 20.363.476.812.021 Francs Congolais;

- Rétrocession de $25 \%$ et de $40 \%$ sur la redevance minière : 6.392.372.373.182 Francs Congolais.

Plusieurs facteurs expliquent la faible mobilisation des recettes du secteur minier dans la province du Haut-Katanga, notamment :

- La non-responsabilisation des intervenants;

- Le non-respect de l'Etat de droit;

- La non-participation de la population à la gestion de la rente minière;

- La non-distribution rationnelle de la rente minière;

- Le manque de transparence dans la gestion de la rente minière;

- La faible lutte contre la fraude minière;

- Le clientélisme politique.

Pour récolter les données, nous avons recouru à l'observation directe et à l'analyse documentaire.

\section{A. LA NON-RESPONSABILISATION DES INTERVENANTS}

Tous les intervenants dans le secteur minier (le Président de la République, le Ministre des mines, le Gouverneur de province, le Chef de division provinciale des mines, le Cadastre minier, la Direction de géologie, la Direction des mines, le Service de protection de l'environnement minier ainsi que toutes parties prenantes) devraient exécuter loyalement leurs tâches.

Le changement de nature de la mission de l'Etat vers une fonction de régulation comptant désormais sur les entreprises pour adhérer, de leur propre initiative, à des standards de développement social, laisse entendre que les entreprises minières définissent librement leur degré de responsabilité sociale. Effectivement, celles-ci viennent parfois combler les lacunes administratives fréquentes dans les pays à faible gouvernance comme la RDC. Si les entreprises occidentales sont parvenues à mettre en place leurs propres standards de gouvernance d'entreprises; les entreprises chinoises, a contrario, ne disposent pas des standards tant pour le développement social, la protection de l'environnement que le respect des Droits de l'Homme, en particulier ceux des communautés locales.

Par ailleurs, la perception juridique chinoise de la responsabilité sociale et environnementale des entreprises dans leur pays est restée très ambiguë. L'irresponsabilité actuelle des entreprises minières chinoises dans la province du Haut-Katanga n'est peut- être pas 
surprenante d'autant plus qu'en Chine même, il existe une profonde incompréhension sur la gouvernance des entreprises, en particulier sur la responsabilité sociale. Cette incompréhension dérive probablement de l'incapacité du gouvernement chinois de mettre sur pied des standards sur l'éthique de ces investissements à l'étranger ainsi que d'un mécanisme susceptible d'assurer que celles-ci se conforment aux politiques cadrant avec les objectifs de la coopération Chine-Afrique.

S'agissant de la forte restriction que connaissent les droits des ouvriers, elle résulte à la fois du capitalisme non réglementé chinois, du caractère faible de ces capitaux investis, mais aussi, évidemment, de l'ignorance de la législation du travail de la RDC par les employeurs chinois et leurs employés. Les travailleurs employés dans les entreprises de moindre taille voient leur traitement empirer. Il est vrai que les conditions de travail dans l'industrie minière nécessitent une plus grande amélioration que dans d'autres entreprises, mais les atteintes répétées portées contre l'intégrité physique des travailleurs dans la province du Haut- Katanga est sans doute une importation chinoise. Dans les entreprises où les libertés syndicales sont exercées, les restrictions des droits des travailleurs prennent une forme moins forte et tendent vers leur amélioration, car il existe un espace de dialogue entre employeur et travailleurs. Ce qui n'est pas le cas des entreprises chinoises.

Il est parfois important de relever que certains employeurs chinois font obstruction aux agents locaux itinérants, chargés de s'assurer que les droits des travailleurs sont observés. Tout cela est bien sûr, dû au dysfonctionnement du système, à la mauvaise administration de la justice sociale et aux bradages des droits des ouvriers par les agents publics locaux en proie à la corruption galopante, compte tenu de la précarité des conditions de travail. Assujettis régulièrement au versement du per diem lors des visites des agents publics, les employeurs chinois perçoivent très mal le travail des agents locaux qualifié de rançonnement. Ces pratiques doivent cesser en vue de valoriser le travail des services de l'Etat et de mieux protéger les droits des travailleurs. Pour ce faire, l'Etat congolais devra mettre des frais de fonctionnement à la disposition de ses services de sorte que, les agents itinérants cessent de rançonner les investisseurs et, par conséquent, de rendre négatif leur travail. La sensibilisation des employeurs chinois est, certes, inévitable pour la promotion globale des droits des ouvriers.

La responsabilité sociale des compagnies minières trouve son fondement dans les normes internes et internationales. Sur le plan interne, l'article 69 point g du code minier de 2002 de la RDC établit cette responsabilité. Le prescrit de cette disposition impose que tout requérant d'un permis d'exploitation joigne à sa demande, entre autres, « le plan pour la contribution du projet au développement des communautés environnantes ». Ce plan permet d'une part au gouvernement, et d'autre part à la communauté concernée, d'évaluer la contribution du projet minier au développement durable de la communauté sur laquelle se déploient ses activités. 
Ce plan fait partie intégrante de l'Etude d'Impacts Environnementaux et Sociaux prescrite par l'article 445 du règlement minier $^{1}$, qui prévoit que la communauté concernée participe aussi, au travers d'un débat sur les résultats définis, au volet social du projet. Ces études permettent d'établir clairement les cahiers des charges des entreprises concernant leur contribution dans la réduction de la pauvreté, notamment la création d'emploi ainsi que la construction des infrastructures sociales de base : écoles, hôpitaux, adduction d'eau potable, électricité, etc.

L'implication et la contribution des entreprises minières dans ces besoins sociaux constituent la responsabilité ou, mieux, les obligations sociales des compagnies.

Cependant, nonobstant l'existence de ce cadre réglementaire, les entreprises minières en général et, en particulier, celles chinoises implantent anarchiquement leurs usines et comptoirs d'achat. Les Etudes d'Impacts Environnementaux et Sociaux, pourtant rendues obligatoires par la législation minière ne sont pas rendues publiques. Parfois, elles ne sont même pas effectuées.

Sur le plan social, l'impact des activités minières des entreprises chinoises est mal perçu par les communautés locales. A Luisha, localité située à $75 \mathrm{Km}$ de la ville de Lubumbashi, où se trouvent basées deux entreprises chinoises, Corporate Mining International Congo (CMICO) et Compagnie Minière de Lubumbashi (COMILU), l'opinion générale sur l'impact social est très négatif. Cette localité vit dans le noir et manque d'eau potable. L'implantation des entreprises chinoises dans cette contrée et les promesses tenues par ces dernières, ont suscité de nouvelles perceptives d'amélioration de condition d'existence, en l'occurrence, l'accès aux besoins sociaux de base : eau, électricité, écoles etc.

Des années après leur installation, aucun aspect de la localité n'a connu un début d'amélioration. L'accès à l'eau potable, l'électricité et les écoles pour des milliers d'enfants demeurent la préoccupation quotidienne de ses habitants. Les deux entreprises CMICO présentes depuis 2004 se sont contentées de forer l'unique borne fontaine située à l'entrée de l'usine au profit des ouvriers. Et pourtant, l'article 69 point g ci-haut cité assujettit tout projet minier à la contribution au développement des communautés environnantes.

Face à cette difficulté permanente d'accès à l'eau potable, certaines familles en quête de cette denrée rare recourent à la clandestinité, et ce, même avec la complicité des gardiens de l'entreprise CMICO pour se procurer de l'eau de la borne fontaine.

La protection de l'environnement occupe indéniablement une place de choix dans la législation minière de la RDC. C'est dans ce cadre que le code minier et son règlement subordonnent l'octroi des droits miniers à l'obtention préalable de l'approbation des études d'impacts environnementaux (EIE), d'un plan de gestion environnemental du projet minier (PGEP), ainsi qu'un plan d'atténuation et de réhabilitation (PAR) auprès des services techniques compétents. La pertinence de ces études vise, d'une part, la mise en œuvre des me-

1 L'esprit et la lettre de cet article sont complètement bafoués car jusqu'à ce jour, la population ignore le contenu de l'accord et n'a pas encore été consultée alors que tout semble être prêt pour la construction de l'usine. 
sures de prévention relatives à la protection de l'environnement et, d'autre part, à la mise en place de mesures d'atténuation et de réhabilitation des risques de dégradation de l'environnement après la fermeture du site minier.

Il est discutable d'aborder l'analyse des défis liés à la dégradation de l'environnement par les entreprises chinoises au Katanga dans un contexte où aucune d'elles n'a mené les études requises par la législation de la RDC, ni, encore moins publié les résultats desdites études. La réalisation préalable de ces études aurait exigé que ces compagnies prennent des mesures de manière à limiter les impacts négatifs majeurs de leurs activités sur l'environnement et sur les communautés locales. Bien que la législation minière en vigueur en RDC fasse de ces études un préalable, sinon une obligation, pour la jouissance des droits ou titres miniers, notamment, tels que précisés dans le code minier: le Permis de Recherche, le Permis d'Exploitation, le Permis d'Exploitation de petite mine et le permis de recherche des rejets, lesquels sont constatés par le Certificat de Recherches, le Certificat d'Exploitation, le Certificat d'Exploitation de Petite Mine et le Certificat d'Exploitation des Rejets, ces études ne sont généralement pas effectuées, ni vérifiées.

A Lubumbashi, à Likasi, et leurs environs, la construction des unités industrielles de traitement de minerais et l'installation des comptoirs d'achat et de dépôts de minerais appartenant aux entreprises chinoises installées dans les quartiers résidentiels, engendrent des risques réels pour les habitants desdits quartiers. Les règles scientifiquement indiquées en la matière, ainsi que la réglementation minière relative à l'entreposage, au transport et à la commercialisation des produits miniers sont systématiquement négligées. Certaines principales avenues des villes citées ci-dessus, servent à l'installation des comptoirs d'achat et à l'entreposage des minerais. Ainsi, l'absence d'études environnementales et la fermeture illégale des entreprises minières chinoises traduit l'absence de l'évaluation des dommages causés sur l'environnement d'une part, et celle des mesures susceptibles non seulement de réparer les dommages probables causés sur l'environnement, mais aussi d'en limiter les effets d'autre part.

\section{B. LE NON-RESPECT DE L'ETAT DE DROIT}

Les entreprises minières sont tenues d'observer les lois et régulations des pays où elles opèrent. Les obligations légales auxquelles elles sont soumises sont prescrites dans les normes internes de la RDC. Il s'agit de l'article 2 du code minier, de l'article $1 \mathrm{er}$ du code de travail, de la réglementation des sociétés commerciales en RDC, de la loi nº9/001 du 10 janvier 2009 portant protection de l'enfant. D'après le rapport RAID, le Ministère du Commerce chinois à Pékin a affirmé que les entreprises chinoises en RDC devaient observer les lois et régulations du travail locales et remplir leurs obligations et responsabilités sociales.

La Constitution de la République Démocratique du Congo considère toute personne ayant moins de 18 ans comme enfant. Tenant compte de sa vulnérabilité, le code du travail en vigueur en RDC le protège suffisamment et qualifie d'illégal le travail qui dépasse ses capacités mentales et physiques et celui des enfants dans les mines et carrières. 
Affirmant sa volonté de garantir et de protéger les droits des enfants, la RDC a mis sur pied, en mi 2009, un cadre normatif dénommé « code national sur les droits de l'enfant».

La convention $n^{\circ} 102$ de l'OIT, ratifiée par la RDC, considère le travail des enfants dans les mines et carrières comme « une charge lourde » sinon une des pires formes du travail pour eux.

L'obligation de protéger les enfants est encore loin d'être atteinte. Cette obligation incombe en premier lieu au pays hôte, en l'espèce l'Etat Congolais. Cependant, l'incapacité de celui-ci ne soustrait l'Etat d'origine des entreprises de son obligation que si celles-ci impliquent les droits de l'homme dans leurs sphères d'actions.

Pour le moment, les droits de l'enfant sont loin d'être protégées en RDC en général et à Lubumbashi en particulier et ce, malgré l'existence d'un cadre normatif presque complet. Les faits ci-dessous en disent plus.

Les résultats des études effectuées par les ONG laissent entrevoir que sur 200 milles mineurs artisanaux employés dans ce secteur, $40 \%$ sont des enfants de moins de 15 ans $^{2}$. Des actions de sensibilisation déployées à cet effet ont amené le gouvernement à durcir des mesures interdisant l'accès des enfants aux mines et carrières artisanales. Cependant, faute d'apporter des réponses aux causes profondes de ce fléau et de propulser ou de stimuler l'engagement de tous les acteurs (collectivité, entreprises privées, autorités locales et organisations locales), ces mesures n'ont pas produit les résultats escomptés.

En revanche, des milliers d'enfants continuent à être exploités dans les mines et carrières artisanales dans le Haut-Katanga. Dans les mines et carrières de Kabunji, Mubambe, Shamitumba, Kamatanda, Kolomoni, Kisakala, Kasonga, Kateketa, Hewa Bora, Mbola pour ne citer que celles-là, les enfants, certains constitués en petits groupes, les autres associés à leurs familles biologiques sont contraints à ces travaux, afin de survivre. Dans toutes ces mines et carrières, les enfants passent de longues heures à concasser des minerais, à transporter de lourdes charges, à tamiser et à laver des minerais dans l'eau, et parfois à extraire des minerais, tout en respirant les poussières nocives et, souvent en présence de substances minérales dangereuses (radioactives). Quand ils participent à toutes ces opérations, leur sécurité et leur santé sont particulièrement menacées.

Dans la carrière de Kamatanda, une carrière d'exploitation de cuivre située à $3 \mathrm{Km}$ de la ville de Likasi, les chercheurs de l'ONG Action Contre l'Impunité pour les Droits Humains ont interviewé quatre enfants mineurs ${ }^{3}$ : Alain, Freddy, Michel et Guelord, âgés de 9 à 15 ans. Les trois premiers opèrent en groupe et le travail dans un groupe d'adultes; le travail consiste dans le ramassage et le tri des minerais, le tamisage et, enfin, la vente de la quantité obtenue à la fin de la journée aux négociants.

2 Coordination du Forum de la société civile de la RDC : rapport sur l'examen indépendant des 12 contrats miniers parmi les plus importants du groupe des 60 contrats, Kinshasa, 2007, p.131.

3 Rapport sur les investissements privés et publics dans le secteur minier au Katanga : Bonne gouvernance et Droits de l'Homme, Kinshasa, 2010, p.11. 
Le véritable enjeu c'est de comprendre à qui profite le travail des enfants dans les mines et carrières artisanales dans la province du Haut- Katanga. La chaine opératoire de la vente de minerai artisanal permet de cerner les bénéficiaires réels et indirects du travail des enfants dans les mines et carrières selon le schéma ci-dessous :

- Enfants mineurs artisanaux négociants (acheteurs congolais);

- Comptoirs d'achat des sociétés industrielles de négoce;

- Compagnies multinationales de fabrique.

Sur le plan national, la chaine de vente comporte deux étapes spécifiques. Dans la première étape, les enfants mineurs vendent le minerai aux négociants et dans la seconde, les négociants les revendent aux sociétés de négoce. Ce scénario fait ressortir le fait que les enfants mineurs sont pris au piège de l'exploitation et ce, au profit des sociétés de négoce. Parmi ces sociétés, il y a lieu de relever de manière singulière la responsabilité des entreprises minières chinoises dont le minerai artisanal constitue la véritable source d'approvisionnement destiné au fonctionnement des fonderies disséminées çà et là dans la province. Cette hypothèse peut se vérifier par la multiplicité des comptoirs d'achat de minerais chinois installés dans toutes les cités et villes minières de la province.

Ces entreprises, comme toutes les autres opérant dans la région, n'ignorent pas l'exploitation dont les enfants font l'objet dans les mines et carrières. Pourtant, elles se lancent délibérément dans l'achat des minerais produits artisanalement sans prendre soin d'envisager l'abolition du travail des enfants. Ceci soulève la question fondamentale sur la double responsabilité (active et passive) des entreprises chinoises dans l'abolition du travail des enfants. Elle est active car, en dépit des normes internes et internationales et des efforts pour l'abolition des pires formes de travail des enfants dans les mines et carrières de la province du Haut-Katanga, ces entreprises exploitent les enfants.

Cette responsabilité est également passive dans la mesure où, en achetant ces minerais, les entreprises (comptoirs) encouragent le travail des enfants dans les mines. Ceci rend vain les efforts des ONG qui militent pour l'abolition du travail des enfants dans les mines et carrières.

A en croire certains employés des entreprises minières, les conditions de travail dans leur entreprise sont quasiment inhumaines et exigent que les autorités gouvernementales en limitent les effets, sous peine de non-assistance à personne en danger. Ils déplorent la dégradation grandissante des relations contractuelles, notamment l'absence des contrats régulièrement conclus avec leur employeur; et, de facto, l'insécurité sociale permanente qui caractérise le travail, la modicité des salaires, l'inexistence des allocations familiales, de logement et autres avantages sociaux, la non-majoration des heures supplémentaires, le non-respect de l'affectation des employés, le non-respect de la dignité humaine des employés caractérisée par des injures intempestives, le manque de transport décent. Tout cela sans aucune possibilité offerte aux employés de négocier avec leur employeur.

Contrairement aux pratiques observées au sein des diverses compagnies minières, en l'occurrence les compagnies chinoises en RDC en général, et dans la province du Haut- Ka- 
tanga en particulier, les relations contractuelles entre ouvrier et employeur sont protégées, voire organisées par la législation du travail de la RDC ainsi que les normes internationales du travail. Le code du travail consacre les droits des ouvriers tels que le droit de négocier et de conclure un contrat écrit à durée déterminée ou indéterminée; de voir son contrat être commué en contrat à durée indéterminée en cas d'engagement du jour au jour dépassant 22 jours sur une période de deux mois; de s'affilier au syndicat de son choix; de gagner une rémunération décente; de bénéficier des conditions de travail et d'hygiène adéquates; de bénéficier de la protection sociale (d'affiliation à l'institut National de Sécurité Sociale, d'un logement décent, d'une prise en charge médicale familiale, etc.)

Qu'à cela ne tienne, bon nombre d'ouvriers se voient régulièrement victimes d'abus de leurs droits. L'essentiel de la main-d'œuvre employée est journalière c'est-à- dire non liée par un contrat de travail alors qu'elle preste des années durant au-delà de 22 jours. De même, ceux qui sont liés par un contrat avec toutes les autres entreprises se voient privés des avantages sociaux y afférents (logement ou indemnité de logement, transport, prise en charge médicale familiale, scolarisation ou indemnités etc.). La plupart des employeurs dans ce secteur en général et chinois en particulier abusent de la situation économique et politique faible du pays pour imposer à leurs ouvriers d'autres types de relations de travail.

Généralement, ces relations sont basées sur la loi du plus fort, dans la négligence totale des droits de l'ouvrier, tels que décrits ci-haut. Nous pensons notamment au droit de négocier et de conclure un contrat écrit; de s'affilier au syndicat de son choix; de gagner une rémunération décente; de travailler en toute sécurité, dans des conditions d'hygiène adéquates; de bénéficier de la protection sociale, etc. Les deux dernières conditions requièrent l'adoption des mesures préventives par l'employeur afin de réduire les cas d'accidents de travail et d'éventuels impacts sur l'environnement. Pour ce faire, l'employeur est invité à former les employés sur les risques de leurs activités, sur leur santé et leur sécurité, ainsi que par rapport à la dégradation de l'environnement; par exemple : ne pas manipuler le produit toxique sans tenue appropriée, ni verser de l'acide dans la rivière.

Cependant, de telles mesures sont loin d'intégrer les politiques sociales et environnementales de la plupart des entreprises chinoises. Visiblement, les entreprises chinoises ne se préoccupent guère des normes requises en cette matière. De plus, l'augmentation des cas d'accidents de travail reste bien évidente.

S'il est vrai que la plupart des entreprises minières chinoises utilisent une grande partie de la main-d'œuvre locale, il est cependant évident que les conditions requises par la loi ne sont pas réunies dans la plupart des contrats en leur sein. En effet, il est important de relever que rien qu'au cours du premier semestre de l'année 2008 par exemple, le programme de droits économiques, sociaux et culturels a enregistré 22 litiges liés au travail dont 7 dans l'une des entreprises occidentales, et 15 , soit environ $70 \%$ des cas essentiellement dans les entreprises chinoises. Ces litiges portent essentiellement sur les accidents de travail, le refus des employeurs de prendre en charge les ouvriers accidentés, et le licenciement collectif et abusif des travailleurs ayant exprimé la nécessité d'exercer le droit à la li- 
berté syndicale et de conclure un contrat écrit après avoir presté comme ouvrier journalier au-delà de la limite des jours impartie pour cette catégorie d'emploi.

Les entreprises chinoises défient l'autorité de l'Etat, qui consiste à garantir la sécurité et la justice sociale des travailleurs. C'est ici qu'il convient d'invoquer sommairement le rôle et la mission dévolue à l'Inspection du Travail. Bien que l'Inspection du Travail soit chargée de veiller au respect de la législation en matière du travail, il est surprenant de constater que ce secteur relève totalement du seul apanage de l'employeur qui en régule les choses comme bon lui semble. Au lieu d'exercer les prérogatives qui leur sont reconnues par la loi, les inspecteurs du Travail justifient leur inaction par la modicité de salaire, l'interférence politique et autres contraintes rendant leur mission impossible. Certains analystes, par contre, y perçoivent la volonté de certains Inspecteurs du Travail de gagner des contreparties auprès des entreprises.

Pourtant, nul n'ignore que les rapports de force entre employé et employeur sont naturellement disproportionnés; et par conséquent, l'intervention de l'Etat est nécessaire en vue d'en garantir l'équilibre et d'y imposer le respect. L'inaction des services publics responsables du contrôle du travail laisse les ouvriers à la merci des employeurs et souvent sans aucune autre alternative.

\section{LA NON-PARTICIPATION DE LA POPULATION A LA GESTION DE LA RENTE MINIERE}

La population riveraine ne participe pas à la gestion des ressources minières et, par de là, de la rente minière. En effet, le secteur minier devrait intégrer désormais une politique de participation de la population locale à la gestion de la rente minière et contribuer au développement communautaire grâce à la mise en œuvre d'une "stratégie provinciale de développement communautaire" dont l'objectif principal est de contribuer à la construction d'un développement durable viable, en particulier dans les localités minières. Une variante de cette stratégie, "le Programme d'Appui aux Communautés Locales" (PACL) devrait offrir une approche provinciale intégrée du développement communautaire orientée sur le déploiement d'un processus de planification participative qui permet à la communauté d'élaborer son Plan de Développement Local (PDL) ainsi qu'un Plan d'Investissement Annuel (PIA).

Au plan social, la responsabilité des entreprises d'extraction découle de l'impact négatif direct de leurs activités sur les communautés locales et constitue, bien que disproportionnelle, une sorte de compensation ou de réparation résultant des préjudices causés aux communautés locales. En d'autres termes, il s'agit de la participation des entreprises à la réalisation des projets qui promettent le développement social des communautés directement affectées : construction d'infrastructures sanitaires, scolaires, eau et électricité, etc. Elles sont différentes des actions volontaires qu'une entreprise peut réaliser en faveur des communautés, et des obligations fiscales qui, elles, sont des redevances minières versées au trésor public. 
Le déplacement des populations autochtones ne doit être autorisé que lorsque, à la suite d'un processus de consultation, la communauté a donné son consentement préalable libre et éclairé à une proposition de projet et aux bénéfices qu'elle compte en tirer;

Ne pas soutenir de projet extractif susceptible d'affecter les populations autochtones sans qu'aient d'abord été reconnus et garantis de manière efficace leurs droits à posséder, contrôler et gérer leurs sols, territoires et ressources;

Reconnaître le droit des femmes en s'engageant à ne pas faire de discrimination.

\section{LA NON-DISTRIBUTION RATIONNELLE DE LA RENTE MINIERE}

Le Code minier de 2002 a privilégié plutôt une logique de redistribution fondée sur le transfert des fonds vers des institutions décentralisées en favorisant une fiscalité qui tient compte du partage des bénéfices avec les communautés locales: $60 \%$ des revenus vont au gouvernement, $25 \%$ reviennent à l'administration de la province où se trouve le projet et enfin $15 \%$ sont alloués à la ville ou le territoire dans lequel s'opère l'exploitation. Les $25 \%$ et les $15 \%$ devraient être affectés exclusivement à la réalisation des infrastructures de base d'intérêt communautaire.

En dotant les entités territoriales locales d'une partie des revenus issus de l'exploitation, ce Code minier a laissé présager une avancée significative en termes de politiques sociales, cherchant ainsi à rompre avec des décennies plutôt caractérisées par la monopolisation des revenus par le pouvoir central. Cependant, en pratique, toutes ces dispositions demeurent lettres mortes et la rente minière n'est pas rationnellement distribuée.

Il est à noter que le rapatriement de $40 \%$ des recettes d'exportations par les entreprises minières relève d'une obligation légale à laquelle les entreprises minières ne peuvent échapper.

En effet, il sied de rappeler que l'actuel code minier est l'œuvre des institutions financières internationales notamment la Banque Mondiale qui a pratiquement imposé ce texte en faveur des investisseurs et qui, au final a plus desservi l'Etat congolais car l'ayant négocié en position de faiblesse.

Le manque de respect des dispositions de ce code dont fait montre les investisseurs, soulève un certain nombre de problèmes, notamment la constitution de la classe moyenne congolaise tant espérée par les autorités congolaises à travers les retombées financières de l'exploitation minière ne relève plus que de l'utopie car les mines ont beaucoup plus appauvri qu'elles n'ont amélioré la vie des populations. Peu avant le débat sur le rapatriement de $40 \%$, le Gouvernement congolais est engagé dans un autre combat avec les miniers sur l'application de la Loi N ${ }^{\circ}$ 17/001 du 08 février 2017 fixant les règles applicables à la soustraitance dans le secteur privé contre laquelle une réticence virulente est manifestée par les entreprises minières invoquant toutes sortes de raisons. Au regard de ce qui précède, tout porterait à croire que les investisseurs évoluant dans le secteur minier n'auraient pas la volonté de respecter les lois de la République démocratique du Congo et seraient très loin de respecter une des obligations essentielles qui incombe à tout investisseur étranger à savoir 
favoriser le développement du pays hôte, car bénéficiant des facilités et de la protection de ce dernier.

La partie congolaise apporte à travers son entreprise Gécamines les droits et titres miniers couvrant les gisements cupro-cobaltifères estimés à 10.616.070 tonnes de cuivre dont 6.813.370 tonnes de cuivre en ressources certaines, et environ 626.619 tonnes de cobalt; et en tonnage restant à déterminer, toutes autres substances minérales valorisables. (Article 4 et annexe de la convention);

Tandis que la partie chinoise, à travers ses entreprises China Railway Group Limited et Sinohydro Corporation, s'emploie à mobiliser les ressources financières pour la réalisation de la joint-venture, elle s'engage à prêter à la Gécamines les fonds nécessaires, en prêt remboursable à $70 \%$ avec un taux d'intérêt de $6,1 \%$.

Eu égard à ce qui précède, les parties susmentionnées se partagent dans la société à créer les actions sociales de la manière suivante : le groupe d'entreprises chinoises détiendra $68 \%$ d'actions, et la Gécamines, entreprise publique congolaise, $32 \%$ (voir article 3.1.4).

La lecture de la convention et de la pratique du droit commercial congolais révèle que l'apport du consortium chinois est un apport en industries. Soulignons que les apports en industrie ne peuvent donner lieu qu'à des actions hors capital appelées « parts des fondateurs ou «parts des bénéficiaires » et non à ces actions hors capital émises en vue de rémunérer certains services rendus ou démarches accomplies par les fondateurs ${ }^{4}$.

S'agissant de la joint-venture, celle-ci se présente de la manière ci-dessous :

Tableau $n^{\circ} I I I .3$ : Gécamines joint-venture :

\begin{tabular}{|l|l|l|}
\hline Sociétés de Joint-venture & $\begin{array}{l}\text { Pourcentage de la Géca- } \\
\text { mines }\end{array}$ & $\begin{array}{l}\text { Pourcentage de la Société de Joint- } \\
\text { venture }\end{array}$ \\
\hline Tenke Fungurume Mining & $17,5 \%$ & $82,5 \%$ \\
\hline Boss Mining & $20 \%$ & $80 \%$ \\
\hline Ruashi Mining & $20 \%$ & $80 \%$ \\
\hline Anvil Mining & $20 \%$ & $80 \%$ \\
\hline
\end{tabular}

Source : Tableau élaboré par les soins de l'auteur.

La lecture de ce tableau atteste le déséquilibre dans la signature de ces joint-ventures et la non- distribution rationnelle de la rente minière.

4 PHAZA-NIANGA di MAZANZA, Guide pratique des sociétés commerciales au Zaïre, Kinshasa, 1988, p.36. 


\section{LE MANQUE DE TRANSPARENCE DANS LA GESTION DE LA RENTE MINIERE}

La transparence est un moyen permettant d'atteindre des objectifs plus larges. Un certain niveau de transparence est une condition nécessaire à l'exercice de la responsabilité, car ceux qui cherchent à contrôler la responsabilité du gouvernement doivent être en mesure de suivre l'action réelle de ce gouvernement. La transparence contribue également à la bonne gouvernance et constitue un obstacle à la corruption.

Dans la perceptive de l'essor de l'exploitation des ressources minières et compte tenu des conséquences engendrées sur l'environnement et de la problématique de l'accès à la justice économique des communautés locales des pays en voie de développement, la transparence s'avère l'outil approprié, sinon la clé, susceptible de remédier à la gestion de la rente minière.

C'est dans ce cadre qu'il convient de situer la pertinence de la dynamique régionale et internationale, traduite sous forme d'initiatives volontaires en vue de promouvoir la transparence dans l'industrie extractive. Plusieurs gouvernements, des grandes compagnies minières, pétrolières, forestières et institutions financières internationales ont souscrit à l'initiative de transparence dans les industries Extractives (ITIE) ${ }^{5}$. Celle-ci, met en évidence le rôle primaire et/ou l'engagement des gouvernements mais aussi celui des compagnies privées à soutenir les efforts des gouvernements engagés dans ce processus.

Le gouvernement de la RDC a adhéré au processus de l'ITIE depuis $2005^{6}$, en vue de parvenir à la publication des paiements, de la gestion, des bénéfices et des dépenses des revenus dérivés du secteur extractif. Conformément au onzième principe de l'initiative, l'engagement du gouvernement congolais, implique celui de toutes les entreprises extractives opérant sur son territoire national. En ce sens l'ITIE apparaît comme le mécanisme par excellence susceptible d'intégrer la transparence dans toutes les compagnies minières opérant en RDC et, par voie de conséquence, de remédier à la gestion de la rente minière.

Cependant, les entreprises minières, comme beaucoup d'autres, ainsi que le gouvernement de la RDC n'ont pas, jusqu'à ce jour, affiché la réelle volonté d'inclure la transparence dans l'exploitation minière. C'est à dire que ni le gouvernement, ni les entreprises minières ne sont parvenus à publier de manière systématique la quantité des redevances minières versées au trésor public à titre d'impôts, taxes douanières, royalties, etc.

Dans ce contexte, l'évaluation singulière du respect des obligations fiscales qui incombent à ces entreprises demeure inconnue. Néanmoins, la recherche effectuée a permis de mettre en exergue des pratiques observables de ces entreprises qui mettent en évidence le fait que ces dernières n'œuvrent pas en toute transparence.

5 Pour en savoir plus : http://www.eitransparency.org/User/File/Keydocuments/factsheetaugust 05 fren ch.pdf, consulté le 22 mars 2016.

6 Pour la RDC, le Rapport du conciliateur indépendant exercice 2007 était en discussion entre les parties prenantes avant sa publication le 09 mars 2010. 
Par exemple, les conclusions du rapport de la Commission économique, financière et bonne gouvernance du Sénat conduite par le professeur Mabi Mulumba laisse entrevoir que les entreprises minières sont loin de mettre la transparence. Le rapport fustige la fraude massive dans le secteur minier de l'ex province du Katanga et révèle que sur 237 entreprises minières opérant dans cette partie, 6 publient leurs statistiques de production, causant ainsi d'énormes préjudices à l'Etat ${ }^{7}$. De toute évidence, ces pratiques sont de la fraude fiscale au regard de l'article 269 , point b du code minier ${ }^{8}$.

D'autre part, le mystère ou l'inaccessibilité des informations sur les activités des entreprises minières ne peut qu'augmenter le doute sur leur transparence et, par conséquent, sur la manière dont elles remplissent leurs obligations légales. Pourtant, ces pratiques amènent la corruption, la fraude fiscale et les malversations récurrentes bien qu'il soit difficile d'en établir les preuves matérielles. Profitant de la faible gouvernance qui caractérise l'administration publique post-conflit, les entreprises minières n'agissent pas en toute transparence. Ce qui ne permet pas aux organisations de la société civile concernées de cerner les véritables acteurs et d'agir en connaissance de cause.

Par ailleurs, certains investisseurs chinois se font passer pour des travailleurs de l'entreprise, tandis que les congolais servent d'interface aux autorités locales, en qualité de responsables d'entreprises ou de comptoirs d'achat de minerais. Parfois, elles sont prises dans le piège de l'escroquerie des complices congolais et payent le prix de leur propre illégalité.

Ces agissements montrent clairement que les investissements dans le secteur minier infirment considérablement les efforts visant à promouvoir la transparence et la responsabilité des acteurs privés et étatiques, et par conséquent, l'exploitation durable des ressources minières.

Cependant, les autorités gouvernementales, se contentent de dénombrer les investisseurs étrangers pour invoquer le «Boom économique » plutôt que de renforcer le contrôle, facteur de la maximisation des recettes fiscales susceptibles de jeter la base d'une croissance bénéfique à l'ensemble de la population. En effet, quand bien même on assisterait à l'émergence d'une volonté des autorités provinciales de limiter les effets dévastateurs des investissements chinois en particulier dans l'exploitation minière, cette volonté serait limitée. La limitation elle-même serait due, en partie, à la course effrénée des intérêts économiques des acteurs étatiques impliqués eux-mêmes dans l'exploitation des ressources minières de la province.

Le contrat entre la RDC et le groupe d'entreprises chinoises a été signé au moment où le gouvernement congolais était en pleine période de revisitassions des contrats miniers (2007) signés au cours de la transition. Que la conclusion de ces contrats ait eu lieu pendant

7 Les extraits de l'adresse du sénateur sont disponibles sur http://www.digitalcongo.net/article/44910, consulté le 12 novembre 2018.

8 « Le titulaire qui exporte les produits marchands des mines est tenu de rapatrier obligatoirement dans son compte national principal tenu en République Démocratique du Congo, $40 \%$ des recettes d'exportations dans les quinze jours à dater de l'encaissement au compte principal prévu à l'article 267 du présent Code». 
une période d'instabilité politique, cela pouvait être, pour le gouvernement issu des urnes à la recherche des moyens financiers pour son programme social, une excuse quant à la nécessité de renégocier lesdits contrats. Force est de constater que pendant cette même période, le même Etat qui soutenait que la plupart des contrats signés pendant la transition l'étaient dans une opacité criante, passe un contrat avec les entreprises chinoises en violation des articles 32 et 33 qui rendent la délivrance des droits miniers obligatoire à un appel d'offre. Le souci du législateur congolais est d'assurer la transparence dans la passation des marchés ou, mieux, dans la signature des contrats miniers.

De plus, l'absence d'un mécanisme d'accès et de divulgation de l'information sur la réalisation des travaux de Joint-Venture demeure pendante. Par exemple, les parties congolaise et chinoise ont signé des avenants modifiant certaines clauses de la convention initiale mais le texte lui-même reste secret.

-La répartition des parts entre parties congolaise et chinoise :

En lisant bien le contrat, on se rend facilement compte qu'il n'y a aucun critère de participation des parts entre associés. Toutefois, le contrat fixe les parts de chaque partie de la manière ci-après : le groupe d'entreprises chinoises $68 \%$, et le gouvernement congolais à travers la Gécamines $32 \%$.

D'après les termes de la convention, la part du lion attribuée au groupe d'entreprises chinoises constitue la garantie offerte à ce dernier afin de recouvrir ses investissements dans les projets miniers et les travaux d'infrastructure ${ }^{9}$. Cette répartition inégale des parts sociales, attire la curiosité sur la nature de l'argent que le consortium chinois va mobiliser pour la création de la Joint-venture. Est-ce un apport ou un prêt?

Le contrat ne donne pas une réponse précise à cette question. Mais en le lisant attentivement, on comprendra tout de suite qu'il s'agit bel et bien d'un prêt. Car l'article 12, 4ème tiret du contrat stipule que : « tous les fonds chinois prêtés aussi bien à la Générale des Carrières et des Mines (Gécamines) qu'à la Joint-Venture seront remboursés avec intérêt, excepté le versement de pas-de-porte de 350 millions de dollars américains dus à la Gécamines à titre de bonus ${ }^{10}$. Deux questions de fond qui ne trouvent pas de réponse dans le contrat sont les suivantes : le consortium chinois va-t-il continuer à être associé au contrat après que son prêt à la Joint-Venture lui aura été remboursé avec intérêt? Si oui, quel sera alors son apport?

9 La création de la joint-venture en plus de 3Milliards qui seront investis pour sa création elle va aussi rembourser les 3 Milliards qui seront consacrés pour la construction des infrastructures (routes, hôpitaux, universités, etc).

10 La gestion de cette somme d'argent semble avoir été opaque. Cette information qui avait justifié la grogne des travailleurs de la Gécamines est disponible http : www.radiookapi.net/index.php? i=53 $\& a=23275$, consulté le 12 novembre 2018 . 
Si le prêt chinois considéré comme apport sera de 3 Milliards, il est cependant malheureux de voir que les biens apportés par la Gécamines en nature, les droits et titres de celle-ci n'ont pas été évalués en argent ${ }^{11}$.

-Des exonérations et exemptions illicites trop élevées :

La législation minière de 2002 en RDC limite le rôle de l'Etat dans le secteur minier à la promotion et à la régulation. Toutefois, elle donne à l'Etat la possibilité de se livrer seul ou en association avec les tiers dans une activité minière sans que la personne morale créée à cet effet bénéficie d'un traitement discriminatoire par rapport aux autres entreprises évoluant dans ce même secteur.

Dans ce contexte, il est inconcevable que la Joint-Venture, qui est une société commerciale, dans laquelle les actionnaires vont tirer des dividendes, jouisse d'une exonération totale. Et surtout que 1' « apport » du consortium chinois sera remboursé avec un intérêt de $6.1 \%$.

Voici comment se présente la portée de ces avantages :

- L'exonération totale de tous les impôts, droits, taxes, douanes, redevances directs et indirects à l'import et à l'export, payables en RDC et ceux liés aux activités minières et au développement d'infrastructures;

- L'exemption des frais et paiements relatifs à la demande, à l'octroi, au transfert et à la cession des droits et titres miniers, des permis de prospection et d'exploitation;

- L'exemption des frais des royalties liées à l'exploitation, etc ${ }^{12}$.

De la garantie du remboursement des prêts chinois

Le remboursement de la dette chinoise est consécutif aux bénéfices des investissements placés dans le secteur minier. Pour ce faire, trois périodes suivantes sont prévues pour le remboursement de la dette : la première période de remboursement minier; la deuxième période de remboursement de la première tranche des infrastructures : et la troisième période commerciale (article 12 de la convention). Pour une bonne compréhension de ces trois périodes de remboursement, il s'avère nécessaire de préciser que, en plus du remboursement avec intérêt de son cout d'investissement estimé à 3 Milliards, la Joint-Venture remboursera égale-

11 L'article 17.3 de la Convention de collaboration entre la RDC et le consortium chinois stipule que « le groupe d'entreprises chinoises s'engage à prêter à la Gécamines les fonds nécessaires pour sa participation au capital social de la Joint-Venture minière et aux augmentations nécessaires de celui-ci».

12 Voir l'article 14, de la convention sur les avantages particuliers pendant les deux premières périodes, pp. $19-20$. 
ment avec intérêt les 6 milliards qui seront investis dans les infrastructures ${ }^{13}$ de base en RDC par les entreprises chinoises ${ }^{14}$.

\section{LA FAIBLE LUTTE CONTRE LA FRAUDE MINIERE}

La lutte anticorruption vise un domaine spécifique à l'origine des faiblesses de la gouvernance. Cependant, il y a la corruption dans le secteur minier dans la province du Haut-Katanga, notamment :

- Le paradoxe scandaleux de grande pauvreté au milieu d'une abondance de richesses minières;

- La traçabilité des revenus continue d'être problématique;

- Les mauvaises pratiques : contrats frauduleux et léonins, comptabilité inexacte, évasion fiscale, contrebande et sous-facturation;

- Les concessions et joint-ventures : contrats octroyés sans appels d'offres et non publiés jusque récemment;

- L'enregistrement d'une fréquence élevée des cas d'évasion fiscale de la part des entreprises minières à travers des contrats miniers secrets, la fusion des entreprises et les acquisitions;

- La falsification des pièces de justification et des documents comptables;

- La surévaluation des investissements par certaines entreprises minières dans le but de diminuer les taxes et impôts dus à l'Etat congolais;

- L'effort de transparence en réponse à des critiques : les contrats revisités publiés sur le site du ministère des mines. La société civile continue à demander la publication du contrat entre la République Démocratique du Congo et la Chine.

A cause de cette avalanche d'indices honteux, la République Démocratique du Congo a pris quelques initiatives de lutte contre la corruption, notamment :

- Les codes minier, forestier et des investissements depuis 2002;

- La Stratégie Nationale de Lutte contre la corruption depuis 2002;

- La loi contre le blanchiment des capitaux depuis 2004;

- La Commission d'Ethique et de Lutte contre la corruption;

- Les reformes de l'Administration publique, des finances publiques, du secteur de sécurité;

- L'adhésion au processus de Kimberley;

- L'adhésion à l'Initiative de Transparence des Industries Extractives;

- La ratification du Protocole de Lutte contre la corruption de la SADC et de la Convention des Nations Unies contre la corruption;

- La Tolérance zéro;...

13 Convention minière entre la Chine et la RDC, article 7.1.

14 Suivant l'esprit et la lettre de la convention du 28 Avril 2007. 
En dépit de cette cargaison des faits de bonne gouvernance, les résultats sont mitigés par :

- Manque de volonté politique;

- Dysfonctionnement des institutions;

- Capture de l'Etat;

- Impunité;

- Guerres des ressources naturelles;

- Tolérance généralisée de la corruption ...

$\bullet$

\section{E. LE CLIENTELISME POLITIQUE}

Les rentes générées servent à financer les réseaux clientélistes proches du pouvoir. Ainsi, il est estimé qu'à partir des années 1980, entre 150 et 400 millions de dollars auraient été détournés des revenus du cuivre et du cobalt, soit près de $30 \%$ de l'ensemble des exportations annuelles. L'appareil productif des entreprises publiques s'est détérioré, conduisant à une faillite, renforcée par les décisions de gestion économiques peu adaptées.

La production de cuivre de la GECAMINES s'est ainsi dramatiquement effondrée depuis la fin des années 1980, passant de 500000 tonnes par an, à 26000 tonnes en 2008 et 7 400 tonnes en 2009. En Juin 2009, la dette cumulée de la GECAMINES fut estimée à 1750 millions de dollars9, et ses pertes évaluées entre 15 et 20 millions par mois ou 167 millions de dollars par an sur les 16 dernières années. Son chiffre d'affaire mensuel en 2009 fut évalué à 4 millions de dollars pour des charges totales avoisinant de 20 millions de dollars. Le poids de ces dettes rend difficile le paiement des salaires de ses 13.000 employés, mais également fournir les ressources nécessaires au fonctionnement des 96 écoles et 7 hôpitaux dont elle a la charge. Leur effondrement a conduit à une désarticulation économique et sociale à proximité des sites d'exploitation.

Aujourd'hui, la Gécamines vit principalement de la vente des permis d'exploration et d'exploitation dont elle dispose et des revenus issus des accords de partage de production dans le cadre de ses partenariats avec des entrepreneurs privés, qui lui aurait rapporté autour de 640 millions de dollars en 2008. Il s'agit cependant d'une stratégie de court terme, la compagnie ne détenant plus, en 2008, qu'entre 44 et 48 permis de recherches, mais qui sembleraient être de faible valeur sur le court terme, ses meilleurs sites ayant déjà été mis en exploitation dans le cadre de divers partenariats.

Sur le plan politique, la province du Haut-Katanga a connu, dans trois ans, trois personnalités politiques (2015-2018) en l'occurrence : le Commissaire Spécial Félicien Katanga, le Gouverneur Jean-Claude Kazembe Musonda et le Gouverneur Célestin Pande Kapopo. La succession du premier Gouverneur nous a révélé le clientélisme politique entre le pouvoir central et les provinces en général et celle du Haut-Katanga en particulier. En effet, le mode de désignation des Gouverneurs des Provinces par le pouvoir central et le respect du mot d'ordre par les Députés provinciaux lors des élections de ces derniers, font que les Gouverneurs soient à la merci du pouvoir central. Dans le cas contraire, le même pouvoir 
central monte des stratégies pour vous faire partir. Ainsi, au lieu de servir le peuple, le Gouverneur sert prioritairement ceux qui l'ont désigné et propulsé pour garder son fauteuil foulant au pied par ce fait même, l'intérêt général.

\section{CONCLUSION}

Des écueils sans nombre jonchent encore le chemin et le combat intrépides pour réamorcer le réamorcer le réarmement moral des gouvernants et des gouvernés face à la forte mobilisation des recettes du secteur minier. Cependant, l'espoir est grand et l'œuvre en vaut la chandelle. Toutes les parties prenantes (institutions politiques nationales, institutions politiques provinciales, entreprises minières, Inspection du Travail, Cours et Tribunaux, Bailleurs de fonds, communautés locales et société civile) sont appelées à prendre conscience de leurs responsabilités en vue de garantir une gestion efficiente des recettes. Pour ce faire, il serait entre autres indispensable d'envisager des sanctions exemplaires contre les services de l'Etat qui affichent une défaillance dans leurs missions et d'auditer les entreprises minières car la corruption soudoie certaines personnalités politiques et hommes d'affaires influents.

\section{REFERENCES BIBLIOGRAPHIQUES}

1. Coordination du Forum de la société civile de la RDC : rapport sur l'examen indépendant des 12 contrats miniers parmi les plus importants du groupe des 60 contrats, Kinshasa, 2007.

2. Rapport sur les investissements privés et publics dans le secteur minier au Katanga : Bonne gouvernance et Droits de l'Homme, Kinshasa, 2010.

3.PHAZA-NIANGA di MAZANZA, Guide pratique des sociétés commerciales au Zaïre, Ed. Afrique Business consultants association, Kinshasa, 1988.

4. http://www.eitransparency.org/User/File/Keydocuments/factsheetaugust05french.pdf, consulté le 22 mars 2016.

5.Pour la RDC, le rapport du conciliateur indépendant exercice 2007 était en discussion entre les parties prenantes avant sa publication le 09 mars 2010 .

6. http://www.digitalcongo.net/article/44910, consulté le 12 novembre 2018.

7. http://www.radiookapi.net/index.php?i=53\&a=23275, consulté le 12 novembre 2018.

8. Convention de collaboration entre la RDC et le consortium chinois.

9. Convention sur les avantages particuliers pendant les deux premières périodes.

10. Convention minière entre la Chine et la RDC.

11. Convention du 28 Avril 2007. 